Dieses Dokument ist eine Zweitveröffentlichung (Postprint) /

This is a self-archiving document (accepted version):

Thomas Mikolajick, Uwe Schroeder, Stefan Slesazeck

Hafnium oxide based ferroelectric devices for memories and beyond

Erstveröffentlichung in / First published in:

International Symposium on VLSI Technology, Systems and Application (VLSI-TSA).

Hsinchu, 16.-19. April 2018. IEEE. ISBN 978-1-5386-4825-4

DOI: https://doi.org/10.1109/VLSI-TSA.2018.8403848

Diese Version ist verfügbar / This version is available on:

https://nbn-resolving.org/urn:nbn:de:bsz:14-qucosa2-769491 


\title{
Hafnium oxide based ferroelectric devices for memories and beyond Thomas Mikolajick ${ }^{*, * *}$, Uwe Schroeder ${ }^{*}$, and Stefan Slesazeck
}

\author{
*NaMLab gGmbH, Noethnitzer Strasse 64, 01187 Dresden, Germany \\ **Institute for Semiconductor and Microsystems, TU Dresden, 01062 Dresden
}

E-mail: Thomas.Mikolajick@namlab.com

\section{INTRODUCTION}

Ferroelectricity is a material property were a remanent polarization exists under zero electrical field that can be reversed by applying an electrical field [1]. As consequence, two nonvolatile states exist that can be switched by an electrical field. This feature makes ferroelectrics ideally suited for nonvolatile memories with low write energy. Therefore, already in the 1950s first attempts have been made to realize ferroelectric nonvolatile memories based on ferroelectric barium titanate (BTO) crystals having evaporated electrodes on both sides [2]. The success of this approach was hindered by disturb issues that could be solved in the early 1990s by adding a transistor device as a selector [3]. Such a memory is referred to as a ferroelectric random access memory (FeRAM). Since reading of the ferroelectric polarization from a capacitor requires switching of the ferroelectric [1], the information will be destroyed and a write back is necessary. This can be avoided if the ferroelectric is placed inside of the gate stack of a MOS transistor resulting in a ferroelectric field effect transistor (FeFET) [1]. Conventional ferroelectric materials like BTO or leadzirconium titanate (PZT) cannot be placed directly on silicon since unwanted interface reactions will occur. The necessary interface layer together with the space charge region of the transistor device leads to a rather low capacitance in series with the ferroelectric dielectric and consequently results in a strong depolarization field that has destroyed the nonvolatility of the FeFET device for many years and hinters scaling as well [4]. Today FeRAM devices are established on the market [3,5], but are limited to niche application since scaling is hindered by many integration problems associated to materials like PZT.

\section{FERROELECTRICITY IN HAFNIUM OXIDE}

In $2011 \mathrm{~T}$. Boeske has reported the ferroelectricity in hafnium oxide for the first time [6]. This unexpected material property of a material already used in CMOS manufacturing has significantly changed the prospects of integrating ferroelectric properties into CMOS processes. The ability to deposit this novel ferroelectric into a 3-dimensional capacitor [7] can help to overcome the scaling limitations of traditional FeRAM while the low dielectric constant together with the high coercive field of this material can solve the retention issues of the FeFET concept [7]. Table 1 gives an overview about the most important properties of ferroelectric hafnium oxide compared to conventional ferroelectric materials.

TABLE 1. COMPARISON OF IMPORTANT PROPERTIES FOR DIFFERENT FERROELECTRIC THIN FILM MATERIALS

\begin{tabular}{l|l|l|l|l} 
& $\begin{array}{l}\mathrm{SBT} \\
\left(\mathrm{Sr}_{2} \mathrm{Bi}_{2} \mathrm{TaO}_{9}\right)\end{array}$ & PZT & $\begin{array}{l}\text { PVDF- } \\
\text { TRFE }\end{array}$ & $\begin{array}{l}\text { ferroelectric } \\
\mathrm{HfO}_{2}\end{array}$ \\
\hline $\begin{array}{l}\text { coercive field } \mathrm{E}_{\mathrm{C}} \\
\text { in MV/cm }\end{array}$ & 0.05 & 0.1 & 0.5 & $0.8-2$ \\
\hline $\begin{array}{l}\text { Polarization } 2 \mathrm{P}_{\mathrm{R}} \\
\text { in } \mu \mathrm{C} / \mathrm{cm}^{2}\end{array}$ & $15-25$ & $30-60$ & 10 & $30-60$ \\
\hline permittivity & $\sim 200$ & $\sim 1000$ & $\sim 2$ & $\sim 30$
\end{tabular}

The most effective way to produce ferroelectricity in hafnium oxide is to add a few percent of dopants or produce a mixed dielectric of hafnium- and zirconium oxide [8]. For dopant materials like silicon or aluminum, having an atomic radius smaller than hafnium, also an antiferroelectric hysteresis can be obtained.

\section{FERROELECTRIC MEMORIES BASED ON HAFNIUM OXIDE}

The complexity of realizing 3-dimensional structures in ferroelectric capacitors based on PZT and similar materials has hindered the scaling of FeRAM below the $90 \mathrm{~nm}$ node. The ability to deposited ferroelectric hafnium oxide into a 3-dimensional layer may resolve that issue. However, the high coercive field compared to conventional ferroelectrics currently still leads to a limited cycling endurance [9]. Recently it has been proposed that using the antiferroelectric hysteresis combined with an external bias field generated by using electrodes with different workfunctions, much better cycling endurance can be achieved [10]. The high coercive field of hafnium oxide based ferroelectrics is of advantage when integrating the material into a FeFET device. On the one hand, the memory window is proportional to the product of the coercive field and the film thickness [11] resulting in a reasonable memory window even when the ferroelectric film thickness is around $10 \mathrm{~nm}$. On the other hand the high coercive field makes the device robust against the depolarization field that is reduced by the lower permittivity of the ferroelectric but still can sacrifice retention [12]. It has been shown that nonvolatile retention together with fast and low power write operation at low voltages can be achieved [13]. In the FeFET device the endurance is not only limited by the degradation of the ferroelectric, but also charge trapping leads to an early closure of the memory window. Recently it has been demonstrated that endurance outperforming Flash devices can be achieved in a hafnium oxide based FeFET [14].

\section{APPLICATIONS BEYOND MEMORIES}

One of the most critical issues when further scaling down CMOS devices is the limitation of the subthreshold swing to $60 \mathrm{mV} / \mathrm{dec}$, since it limits supply voltage scaling and therefore energy efficiency improvement. About 10 years ago it was proposed that a ferroelectric in series with a properly designed gate insulator can result in a stable negative capacitance that will improve the subthreshold swing below $60 \mathrm{mV} / \mathrm{dec}$ and at the same time improve the on-current [15]. Many questions about stabilizing the normally unstable region of the ferroelectric switching still need to be worked out. Therefore, the potential benefit from such a device has spurred considerable interest from research and industry. All practical devices use ferroelectric hafnium oxide since it is the only known ferroelectric that can be 
seamlessly integrated into an existing CMOS process and that can be scaled to the necessary thickness range [16]. One of the major challenges seems to be the domain structure. When scaling down FeFETs to the nanometer dimensions, abrupt steps in the switching behavior can be observed that can be interpreted as the switching of single domains [17]. It was recently proposed to utilize this effect for the realization of a true random number generator (TRNG) [18]. The so called "von Neumann bottleneck" is a severe issue for further performance enhancement and energy efficiency improvement. Therefore, merging the memory and the logic function could help to drastically enhance the performance of future electronic systems. With hafnium oxide based FeFET, for the first time a transistor based nonvolatile memory cell is available that can be scaled like a CMOS device and operates at reasonable low voltages. Therefore, the memory devices can be distributed within the logic circuits opening up the path for new ways of signal processing. A first example showing a reconfigurable NAND/NOR gate based on one FeFET and one load device was recently shown [19]. Going further, distributed memory cells could be used to store the weights in artificial neural networks (ANN). Even further out, also spiking neural networks will need some sort of memory to realize the synaptic function. Here hafnium oxide based FeFETs can realize a synaptic function [20]. These example demonstrate that having the ability to integrate a ferroelectric into a CMOS process cannot just lead to better and cheaper memories, but also hold the promise to realize a number of new applications in much more efficient implementations. Finally it needs to be mentioned that the nature of ferroelectricity implies that ferroelectric materials are also pyroelectric and piezoelectric. The exploitation of these effects in a fully CMOS compatible manor is only in its very infancy $[21,22]$ and can be used in the field of sensors, actuators and energy harvesting and energy storage.

\section{SUMMARY}

Ferroelectricity is a material property that allows a wealth of different possibilities when integrated into electron devices. Classical ferroelectric materials like perovskites can only be integrated with many drawbacks like complicated processing and most important limited scalability. The discovery of ferroelectricity in hafnium oxide holds the promise to overcome the most severe issues in both capacitor and transistor based memory devices but also enables new ideas were the memory function and the logic function are merged. Moreover, the exploitation of the pyroelectric and piezoelectric properties are still in a very early stage. Figure I illustrates various potential applications of ferroelectric hafnium oxide reported so far.

\section{REFERENCES}

[1] T. Mikolajick, Ferroelectric Nonvolatile Memories, in Ref. Module in Mat. Sc. and Mat. Eng., Elsevier (2016)

[2] J. Merz, and J.R. Anderson, Bell Lab Records 33, 335-342 (1955)

[3] D. Bondurant, Ferroelectrics 112, 273-282 (1990)

[4] T.P. Ma and J.P. Han, IEEE Electr. Dev. Lett. 23, 386-388 (2002)

[5] H. P. McAdams et al., IEEE J. Solid-State Circuit 39, 667 (2004)

[6] T. S. Böscke et al., Appl. Phys. Lett. 99, 102903 (2011)

[7] J. Müller et al., IEDM, pp. 10.8.1 - 10.8.4 (2013)

[8] U. Schroeder et al, Jpn. J. Appl. Phys. 53, 08LE02 (2014)

[9] U. Schroeder et al, Proceedings of ESSDERC, 364-368 (2016)

[10] M. Pešić et al., IEDM, pp. 11.6.1-11.6.4 (2016)

[11] J. Mueller et al., ECS Transactions 69, 85-95 (2015)

[12] J. Mueller et al., ECS J. Solid State Sci. Tech. 4, 5, N30 (2015)

[13] S. Duenkel et al., IEDM (2017)

[14] K. Chatterjee et al., IEEE Elec. Dev. Lett. 38, 10, 1379 (2017)

[15] S. Salahuddin and S. Datta, Nano Lett., 8, 405 (2008)

[16] Z. Krivokapic et al, IEDM (2017)

[17] H. Mulaosmanovic et al., ACS Appl. Mat. Interf. 9, 4, 3792(2017)

[18] H. Mulaosmanovic et al., Electr. Dev. Let. 39, 135 (2018)

[19] E. T. Breyer et al., IEDM (2017)

[20] H. Mulaosmanovic et al., Symp.VLSI Technology, T176 (2017)

[21] M. Hoffmann et al, Nano Energy 18, 154-164 (2015)

[22] M. H. Park et al., Advanced Materials 27,11, 1811 (2015)

\section{ACKNOWLEDGEMENT}

Part of this work was supported by the EFRE fund of the European Commission within the scope of technology development, the Free State of Saxony (Germany), and the German Research Foundation (Deutsche Forschungsgemeinschaft; project MI 1247/11.

\section{FIGURE I: ILLUSTRATION OF VARIOUS POTENTIAL APPLICATION FIELDS FOR FERROELECTRIC HAFNIUM OXIDE}

\section{Sensors /Actuators / Energy harvesting}

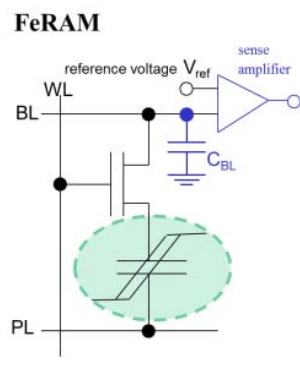

Memory in Logic

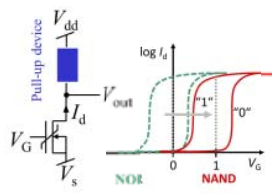

Negative Capacitance FET

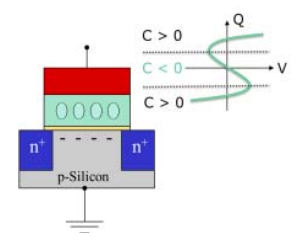

FeFET Memory
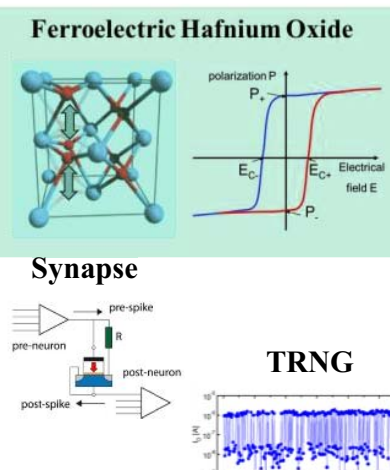

TRNG
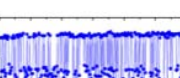
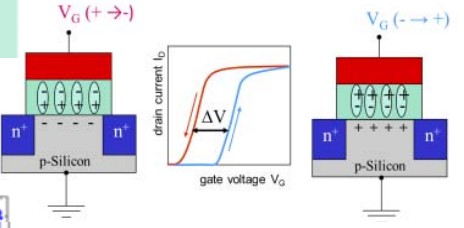